\title{
Dangling-to-Interstitial Oxygen Transition and Its Modifications of the Electronic Structure in Few-Layer Phosphorene
}

\author{
Juan F. Gómez-Pérez,* Julián D. Correa, Cora Bartus Pravda, Zoltán Kónya, and Ákos Kukovecz* \\ Cite This: J. Phys. Chem. C 2020, 124, 24066-24072 \\ Read Online
}

ABSTRACT: In this work, oxidation processes are correlated with the current-voltage characteristics of few-layer black phosphorus obtained by liquid-phase exfoliation. Black phosphorous (BP), a room-temperature p-type semiconductor, exhibits an anomalous switching behavior between 373 and $448 \mathrm{~K}$. The anomalous increase in electrical resistance is explained using a combined spectroscopic and DFT approach. The activation energy for thermally activated electrical conductance was calculated from the current-voltage characteristics and correlated with the oxidation processes. The activation energy for thermally activated electrical conductance in the dangling oxide BP phase was found to be $79.7 \mathrm{meV}, \sim 40$ times lower than that in the

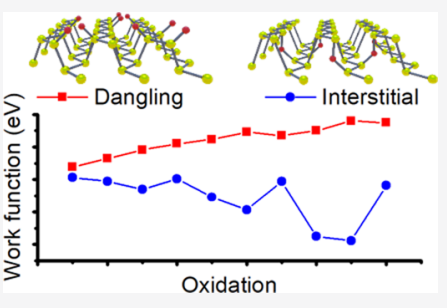
interstitial counterpart. First-principles calculations reveal electronic differences between dangling and interstitial oxides, and electrical resistance measurements reveal a Schottky-to-ohmic contact formation related to the differences in the calculated work function of dangling and interstitial oxides. We propose that this phenomenon can be exploited as a fast, economical method for the evaluation of the oxidation processes in few-layer BP.

\section{INTRODUCTION}

Few-layer black phosphorus has attracted considerable interest since 2014 , as demonstrated by the $700+$ papers published in this field in 2019 alone. This is fueled by the performance limits of the Si-based technology and the intrinsic limitations of graphene. Phosphorene is a stable monoelement twodimensional (2D) p-type semiconductor with potential applications in broadband photoresponse field-effect transistors (FETs) ${ }^{1}$ and sensors (e.g., $\mathrm{NO}_{2},{ }^{2,3} \mathrm{NH}_{3},{ }^{4}$ humidity, ${ }^{5}$ and biomolecules $^{6}$ ). These applications rely on the charge transfer process between black phosphorus and metal electrodes. Thus, a better understanding of these junctions is required for the design of electric devices. ${ }^{7,8}$

Black phosphorus exhibits highly tunable electronic properties between those of graphene and transition-metal dichalcogenides. For instance, the measured electronic band gap of black phosphorus is direct and varies with the number of layers between $0.3 \mathrm{eV}$ (i.e., bulk) and $\sim 2.2 \mathrm{eV}$ (i.e., monolayer), ${ }^{9,10}$ and the carrier mobility of exfoliated materials ( 1000 and $\left.6000 \mathrm{~cm}^{2} \mathrm{~V}^{-1} \mathrm{~s}^{-1}\right)^{10}$ is in the middle between values reported for graphene $\left(15,000-200,000 \mathrm{~cm}^{2} \mathrm{~V}^{-1} \mathrm{~s}^{-1}\right)^{11,12}$ and those for transition-metal dichalcogenides $\left(10-1000 \mathrm{~cm}^{2} \mathrm{~V}^{-1}\right.$ $\left.\mathrm{s}^{-1}\right) .{ }^{13}$ Nevertheless, black phosphorus is easier to oxidize than other $2 \mathrm{D}$ materials. The energy barrier for the formation of the transition state during the oxidation of the black phosphorus monolayer is the lowest (i.e., $0.1-0.5 \mathrm{eV})^{14,15}$ in comparison with other 2D materials (e.g., graphene: 2.2-2.7 eV, ${ }^{16}$ $\mathrm{MoS}_{2}: 1.6 \mathrm{eV},{ }^{17}$ and h-BN: $\left.1.2-2.6 \mathrm{eV}^{18}\right)$. This disadvantage will be an obstacle for the industrial application of black phosphorus unless a deeper understanding of the oxidation of $2 \mathrm{D}$ materials and better control of the process is achieved. ${ }^{19-23}$ It would be highly desirable to avoid repeating the fate of graphene, for which quality (e.g., defects) and standardization issues have emerged as barriers for the industrial application regardless of the academic enthusiasm for the beauty of the science involved.

For black phosphorus, two different approaches have emerged to overcome the oxidation issues: (i) chemical functionalization and passivation and (ii) band gap engineering by native oxides. Recently, several examples of passivation and covalent functionalization of black phosphorus have been published $^{20,24-26}$ to tackle this issue, and improved stabilities of micromechanically exfoliated samples have been achieved at room temperature for evaluation periods of several weeks. However, in situ methods are still required to determine the stability of the passivated/functionalized materials under operating conditions. The band gap engineering by oxidation has been proposed in theoretical studies, ${ }^{21}$ but there is an ongoing debate in the recent literature on the relative stability and characterization of the different types of oxides (see Figure 1) under near-equilibrium conditions. Somewhat contradictory results have been published regarding the stability of planar, surface, and tubular types of oxides, with works pointing to the higher stability of either the planar (Figure $1 \mathrm{~d})^{21}$ or the surface oxide (Figure 1a). ${ }^{27,28}$

Received: July 17, 2020

Revised: October 7, 2020

Published: October 22, 2020 


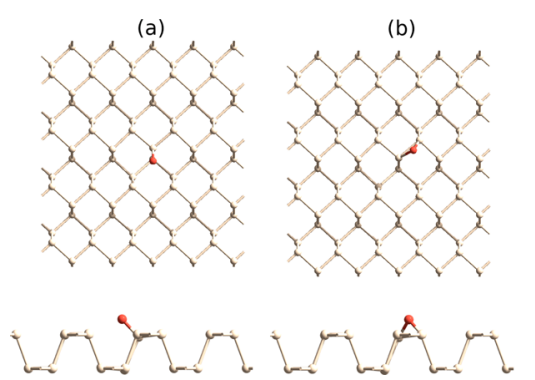

Figure 1. Schematic representation of an oxygen atom at (a) dangling,

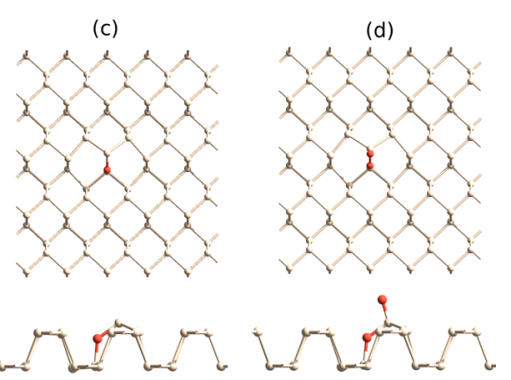

The precise identification of the different types of oxides by XPS has been challenging. This can be seen from different studies that have assigned the same fitted peak at $131.5 \mathrm{eV}$ to the $\mathrm{P}=\mathrm{O}$ (dangling) or the $\mathrm{P}-\mathrm{O}-\mathrm{P}$ (bridging oxides) during the deconvolution of experimental spectra. ${ }^{26,29}$ Moreover, the precise bending energy varies with concentration, and the relative intensities are weak, making the measurement and identification more demanding. Nevertheless, XPS has been successfully used for the evaluation of passivation, which improves the stability of transistors in ambient conditions, for example, by confirming the formation of the $\mathrm{P}-\mathrm{S}, \mathrm{P}-\mathrm{F}$, or the $\mathrm{P}-\mathrm{O}^{-}-\mathrm{NH}_{3}{ }^{+}-\mathrm{R}$ bondings when black phosphorus is functionalized with sulfur, ${ }^{30}$ fluor, ${ }^{31}$ or alkylamines, ${ }^{32}$ respectively.

For pristine exfoliated black phosphorus, the dependence of the electronic band gap on the number of layers is well-known since $2014,{ }^{33}$ and the largest variations of the electronic structure occur between the monolayer, bilayer, and trilayer systems, mainly at the position of the valence band maximum (VBM) ${ }^{33,34}$ In practice, it means that the potential barrier height between exfoliated black phosphorus (p-type, roomtemperature semiconductor) and metal electrodes is highly sensitive to the number of layers in transducer applications. However, even though the oxidation process affects the electronic structure of exfoliated black phosphorus more than the number of layers (the expected expansion of the band gap is larger than $6 \mathrm{eV}^{21}$ ), the performance of phosphorene oxides has been scarcely investigated experimentally for electric devices. $^{35,36}$

In this work, we investigate the formation of different types of oxides and their impacts in contacts between gold and black phosphorus. Measuring the activation energy for thermally activated electric conductance provides a simple, economical, and fast method to evaluate the effects of oxidative processes in few-layer black phosphorus on electric devices. Additionally, our DFT calculations evidence a higher probability for the formation of the dangling oxygen position than for interstitial or bridging positions, with the latter two at degenerated levels. More importantly, relevant electronic differences are demonstrated by the occurrence of different types of oxides in the contact between gold electrodes and oxidized black phosphorus.

\section{METHODS}

Few-layer black phosphorous (BP) flakes were produced with the same protocol as in our previous studies: ${ }^{37}$ for liquid-phase exfoliation with an ultra-sonication bath $(35 \mathrm{kHz}, 120 \mathrm{~W}), 5$ mg of bulk BP crystal purchased from HQ Graphene (the Netherlands) was added to $15 \mathrm{~mL}$ of acetone in a glass vial and closed with a septum cap. The mixture was purged with argon and ultrasonicated for $24 \mathrm{~h}$ at $298 \mathrm{~K}$. The sample was allowed to sediment overnight, and only the supernatant was collected and labeled as BP AsEx. The light absorption of the BP AsEx suspensions is correlated with the degree of exfoliation. From the UV-vis spectra (UV-3600 Plus, Shimadzu), optical band gaps were measured and compared against previous experimental and theoretical results to confirm the identification of the material. Also, the obtained materials were characterized using an FEI TECNAI G2 20 X-Twin transmission electron microscope (TEM) operated at $200 \mathrm{kV}$ accelerating voltage and Raman spectroscopy (Senterra II, Bruker, $\lambda=532 \mathrm{~nm}$ ) as well.

Temperature-dependent current-voltage characteristics were measured with a Keithley 2401 source meter. Four-wire connections were used for the measurements in the voltage range $-0.5 \mathrm{~V}$ to $+0.5 \mathrm{~V}$. For the electrical characterization, the exfoliated sample (BP AsEx) was drop-cast on gold interdigitated electrodes purchased from Micrux technologies (Spain) with a spacing of $5 \mu \mathrm{m}$ between the gold electrodes and a glass substrate (see the Supporting Information, Figure S1). This assembly was placed in a closed-cell (Linkam HFS600E microscopic stage) that allows simultaneous temperature control, gas atmosphere control, Raman spectrometry, and current-voltage measurements. The cell was purged with argon and pre-treated at $398 \mathrm{~K}$ for $30 \mathrm{~min}$ to remove any water and remnant solvent traces. After the pre-treatment, the sample is cooled to $298 \mathrm{~K}$ and the measurements were made in an argon flow atmosphere.

The geometrical structures for each phosphorene oxide are evaluated using the density functional theory (DFT), as is implemented in SIESTA. ${ }^{38}$ For that purpose, conservative norm pseudopotentials are used in addition to localized atomic orbitals, specifically, the polarized double- $\zeta$ base. For the exchange and correlation functional, we used the GGA approximation. ${ }^{39}$ All structures are relaxed until the forces on each atom fall under $0.02 \mathrm{eV} / \AA$. The calculation was done for a supercell of $5 \times 4 \times 1$, ensuring a minimal vacuum space between perpendicular images of $10 \AA$. The total energy convergence is achieved by using a Monkhorst-Pack grid of $2 \times$ $2 \times 1$ to sample the Brillouin zone. For calculating the total density of states (DOS), we employed a dense k-point grid of $51 \times 51 \times 1$. The work function was defined as

$$
\phi=E v-E f
$$

where $E v$ is the vacuum energy, and $E f$ is the Fermi energy level. To get the vacuum energy, we employed the Macroave SIESTA tool.

The oxide occupation percentage is defined as the ratio of oxygen atoms needed to form an oxide (dangling: 1, interstitial: 1, dangling+interstitial: 2) by the number of available sites. In the case of the $5 \times 4 \times 1$ supercell, 80 
phosphorus atoms provide 40 available sites to be occupied by oxygen atoms.

\section{RESULTS AND DISCUSSION}

Several models of phosphorene oxide (BPox) have been proposed $^{15,21,26,28}$ including the following metastable oxides: dangling oxygen, when the oxygen atom is chemically bonded on the surface to one single phosphorus atom; oxygen bridge, for the oxygen atom chemically bonded to two consecutive phosphorus atoms, and interstitial oxygen when the oxygen atom penetrates the lattice. These models predicted BPoxs to be stable in both stoichiometric and nonstoichiometric configurations, $^{15}$ and they have shown that phosphorene oxidation can lead to the formation of a new family of phosphorus oxides where the band gap can be modulated by varying the oxygen concentration in the lattice. ${ }^{21}$ The novelty of the present approach is that we combine both experimental and first-principles results to study the oxidation-induced changes in the work function of phosphorene.

Experimentally, the characterization of the BP nanoflakes shows a hydrodynamic size of $250 \pm 60 \mathrm{~nm}$ (DLS, Malvern, Zetasizer-NanoZS) in agreement with TEM images (Figure $2 \mathrm{a}$ ) and two absorption edges in the UV-vis spectrum (Figure
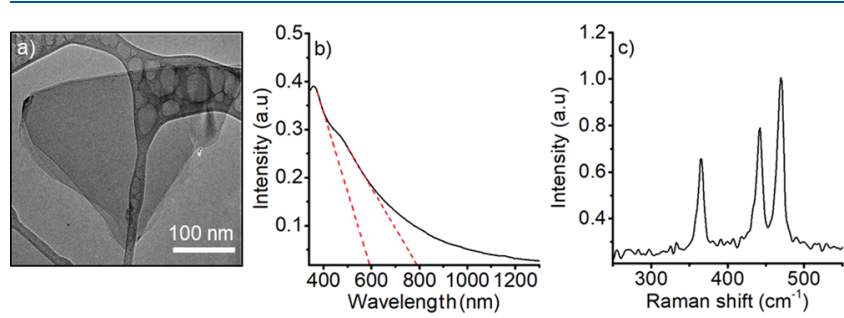

Figure 2. (a) TEM image of a few-layer black phosphorus flake; (b) UV-vis-NIR spectra for the BP AsEx sample suspended in acetone, with dashed lines indicating the observed absorption edges, and (c) characteristic Raman spectra for the drop-cast flakes exhibiting the vibrational modes $\mathrm{A}_{\mathrm{g}}^{1}\left(365.5 \mathrm{~cm}^{-1}\right), \mathrm{B}_{2 \mathrm{~g}}\left(442.2 \mathrm{~cm}^{-1}\right)$, and $\mathrm{A}_{\mathrm{g}}^{2}(470.2$ $\left.\mathrm{cm}^{-1}\right)$.

$2 \mathrm{~b})$ : the first corresponding to an electronic band gap of $\sim 1.57$ $\mathrm{eV}$ (i.e., exciton binding energy: $-31.1 \mathrm{meV}$ in acetone ${ }^{40}$ ) and a shoulder at $\sim 600 \mathrm{~nm}$, which corresponds to an optical bandgap of $2.1 \mathrm{eV}$ (Figure $2 \mathrm{~b}$ ) in good agreement with the expected values for monolayers and bilayers, respectively. ${ }^{33,34}$ We can conclude that the obtained suspension is composed of monolayers, bilayers, and up to 4-layer flakes, as previously reported for a similar exfoliation protocol. ${ }^{23,37}$ It is important to recall that quantum confinement effects are only visible for flakes below 20 layers $^{9}$ and that the yield of few-layer black phosphorus is significantly higher and reproducible in liquidphase exfoliation than in micromechanical methods. ${ }^{40}$

The Raman $\mathrm{A}_{\mathrm{g}}{ }_{\mathrm{g}} / \mathrm{A}_{\mathrm{g}}^{2}=0.53$ ratio of $\mathrm{BP}$ AsEx (Figure 2c) indicates slight oxidation and the formation of metastable oxides during sample manipulation and exfoliation. ${ }^{36,41-44}$ However, the identification of the different types of oxides and their impacts on the performance of electric devices is not yet evident. The differences in the electronic properties of metastable BPox are investigated and verified in electrical resistance measurements and computational experiments below.

First, from the electrical resistance measurements, three different processes can be identified for the samples in Figure 3 and Figure S2: (i) at low temperatures (i.e., below $373 \mathrm{~K}$ ), the
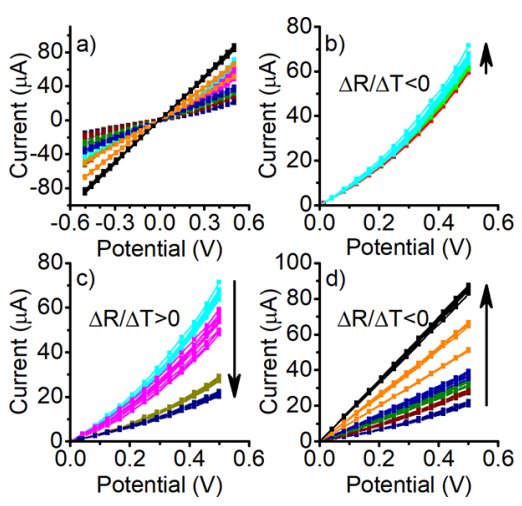

Figure 3. (a) Current-voltage characteristics for acetone-exfoliated black phosphorus in the temperature range 298 to $573 \mathrm{~K}$ in an argon atmosphere. Detailed forward bias in the temperature range (b) 298 to $373 \mathrm{~K}$, (c) 373 to $448 \mathrm{~K}$, and (d) 448 to $573 \mathrm{~K}$. The arrows indicate the direction of increasing temperature.

exfoliated black phosphorus exhibits the typical semiconductor behavior insofar as its resistance decreases with increasing temperature, (ii) between 373 and $448 \mathrm{~K}$, an anomalous behavior is characterized by an opposite trend, typical of ordinary metals (i.e., the electrical resistance increases with temperature, ${ }^{45} \Delta \mathrm{R} / \Delta \mathrm{T}>0$ ); and (iii) the electrical resistance decreases again with increasing temperatures above $448 \mathrm{~K}$, as expected for semiconductors. To our best knowledge, this is the first time that such complex behavior is reported for exfoliated BP. The semiconductor-to-metal transition in 2D materials has been predicted recently for phosphorene, germanene, and stanine computationally. ${ }^{46-48}$ For example, Mounkachi et al. (2018) predicted a semiconductor-to-metal transition in exfoliated black phosphorus after the adsorption of four or more $\mathrm{Li}, \mathrm{Na}$, or $\mathrm{Mg}$ atoms on the surface of the studied supercell ( 8 phosphorus atoms) for $(\mathrm{Li} / \mathrm{Na} / \mathrm{Mg})$-ion battery applications, ${ }^{46}$ but no experimental evidence has been published yet and the discussion of the phenomena taking place during oxidation processes is still lacking.

DFT calculations were utilized to investigate the variations of the electronic structure in comparison with pristine BP and explain the experimental results presented in Figure 3. For pristine phosphorene, we report a band gap of $0.85 \mathrm{eV}$ and a work function of $4.64 \mathrm{eV}$, but after the addition of one single oxygen atom at a dangling, bridging, or interstitial position (Figure 1), slight modifications in the band gap and work function were observed (Table 1). The largest variations were

Table 1. Calculated Electronic Properties after the Addition of One Oxygen Atom to a Pristine Phosphorene Layer Supercell $5 \times 4 \times 1$

\begin{tabular}{lccc}
\multicolumn{1}{c}{ system } & band gap $(\mathrm{eV})$ & work function $(\mathrm{eV})$ & $\mathrm{Eb}(\mathrm{eV})$ \\
BPox dangling & 1.00 & 4.47 & 1.95 \\
BPox bridge & 1.00 & 4.46 & 1.29 \\
BPox interstitial & 1.04 & 4.43 & 1.46 \\
\hline
\end{tabular}

found after the formation of the interstitial oxide followed by the bridging and dangling oxides. The slight decay of the work function supports experimental results on black phosphorus published recently ${ }^{49}$ but also is a common trend for other oxides. $^{50}$

In general, our results suggest that the adsorption of one oxygen atom at the dangling or bridge position does not 
induce appreciable changes in comparison with the lattice of pristine phosphorene, whereas the interstitial oxide produces considerable differences in its surroundings. The binding energy defined as, i.e., $E_{\mathrm{b}}=-\left(E_{\mathrm{BP}}-\mathrm{xO}-\left(E_{\mathrm{BP}}+N_{\mathrm{O}} E_{\mathrm{O}}\right)\right) / N_{\mathrm{O}}$ indicates that the adsorption of one oxygen atom at the dangling position is the most favorable oxide, in agreement with previous work. ${ }^{15}$

The general shape of the total DOS (Figure 4) is conserved for each of the oxygen adsorption site types considered in

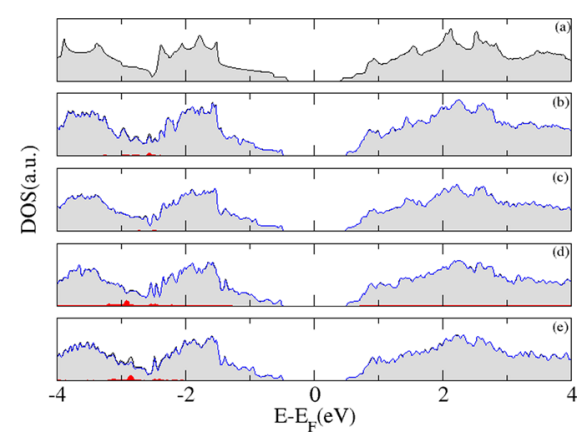

Figure 4. Total (black), oxygen- (red) and phosphorus- (blue) project electronic density of states for (a) pristine phosphorene and phosphorene oxides at (b) dangling, (c) bridge, (d) interstitial, and (e) dangling+interstitial positions after the adsorption of one oxygen atom.

Figure 1. However, some new small Van Hove singularities arise along with all DOS. These singularities are most prominent for oxides with interstitial oxygens, which suggests that the increase in the concentration of adsorbed oxygen could induce great changes in the phosphorene electronic structure and absorption. In conclusion, after the addition of one single oxygen atom, our computational model shows a slight increase in the electronic band gap and a lower work function for all the evaluated types of oxides.

Next, the effect of increasing oxygen concentration was evaluated for three phosphorene-oxide models: dangling, interstitial, and dangling + interstitial oxides. For the models, oxygen atoms are placed randomly in the defined configuration (Figure 5) to reach oxygen concentrations between 1.2 and $19.2 \%$ (see Methods), and these models are used to investigate the dependence of the electronic properties on the oxide concentration.

The computational results presented in Figure 6 reveal striking differences between the work function levels of surface and interstitial oxides at low oxygen concentrations. Surface
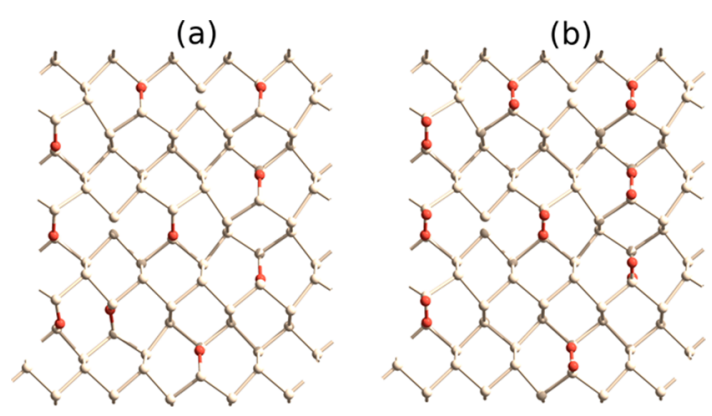

Figure 5. Typical models illustrating the evaluation of the oxygen concentration at (a) interstitial and (b) dangling+interstitial positions. Figures represent an oxygen concentration of 11.1 and $18.4 \%$.
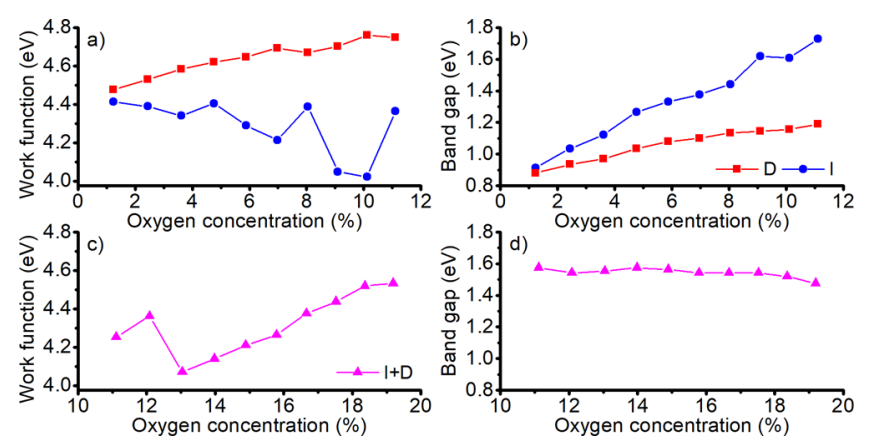

Figure 6. Variations of the (a) work function and (b) electronic band gap at low oxygen concentrations at dangling (D) and interstitial (I) positions. Variation of the (c) work function and (d) band gap at higher oxygen concentration at dangling+interstitial $(\mathrm{D}+\mathrm{I})$ positions.

and planar oxides are characterized by a monotonic increase of the work function with increased oxygen concentration, in support of recently published experimental results. ${ }^{49}$ On the other hand, we show for the first time that the interstitial type is characterized by a decreasing work function and a larger expansion of the band gap. The oscillations in Figure 6a for the interstitial oxide are possibly induced by the random choice of the interstitial site in our model. At higher oxygen concentrations (Figure $6 \mathrm{c}, \mathrm{d}$ ), the difference between the work functions of dangling and interstitial oxides or dangling and Interstitial + Dangling oxides can be larger than $0.5 \mathrm{eV}$, and therefore, these oxides may form different contact types at the heterojunction between gold and exfoliated black phosphorus.

The experimental results in Figure 3 show a Schottky-toohmic transition in the type of contact between gold and black phosphorus at temperatures above $448 \mathrm{~K}$. For junctions between metals and p-type semiconductors, ohmic contacts are formed when the work function of the metal is larger than the work function of the semiconductor. Otherwise (i.e., when the work function of the metal is smaller than the work function of the semiconductor), Schottky contacts are formed. Ohmic contacts are desired for electric devices as they improve efficiency and reduce heat dissipation that may result in poor device lifetimes. ${ }^{51}$ We may conclude that the type of contact between the Au electrode and the few-layer BP-discernible from the shape of the current-voltage characteristic curvesdepends on the differences in the work function, the position of the VBM, and interface states at the heterojunction. This is a practical example of band gap engineering by oxidation as a consequence of the work function differences between the different types of oxides.

Thermogravimetric analysis, differential scanning calorimetry, and in situ Raman spectroscopy measurements presented in our previous work ${ }^{23}$ help to explain the observed results: a broad endothermic process and a large expansion in the $\mathrm{z}$ direction perpendicular to the plane have been proposed as the oxygen endothermic dangling-to-interstitial transition characterized. In this work, the formation of the interstitial oxide is confirmed by the modification of the work function as described by our computational results (Figure 6) and reveals particular trends for each type of metastable oxide.

The activation energy $(E a)$ of thermally activated electric conductance for the data in Figure 3 is presented in Figure 7 as a function of temperature and summarized in Table 2. The electrical resistance was computed from the slope of the 


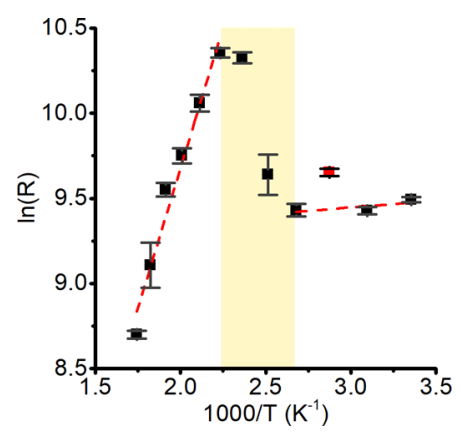

Figure 7. Arrhenius plot for the calculation of the activation energy of thermally activated electric conductance. Black squares represent measurement points and their standard deviation. The red square denotes an outlier, and dashed lines indicate the linear fits according to eq 2. The dangling-to-interstitial transition region is marked in yellow.

Table 2. Activation Energies for Selected Thermally Activated Electric Conduction Processes

\begin{tabular}{lcc}
\multicolumn{1}{c}{ material } & Ea $(\mathrm{meV})$ & ref. \\
few-layer BP oxide (dangling) & 79.7 & this work \\
few-layer BP oxide (interstitial) & 3295 & this work \\
\hline
\end{tabular}

current-voltage curves, and the activation energy was calculated according to eq 2 .

$$
\ln R=\ln R_{0}+\frac{E_{a}}{K_{\mathrm{B}} T}
$$

Here, $R$ is the electrical resistance in ohms, $R_{0}$ is the intercept at $0 \mathrm{~K}, E a$ is the activation energy in $\mathrm{eV}, K_{B}$ is Boltzmann's constant, and $T$ is the temperature in Kelvin.

Equation 2 describes intrinsic processes in the semiconductor: at higher temperatures, more and more carriers are activated getting through higher energy levels; therefore, the transition region (the yellow region in Figure 7) must be explained by an exogenous process. This can be the incorporation of oxygen atoms into the lattice and the formation of a new type of oxide with interstitial oxygen atoms.

Monotonic decay of carrier mobility and $I_{\mathrm{ON}} / I_{\mathrm{OFF}}$ ratios have been identified as a consequence of oxidation in ambient conditions for 21 days. Typically, exfoliated black phosphorus carrier mobility and $I_{\mathrm{ON}} / I_{\mathrm{OFF}}$ ratios range between $500-1000$ $\mathrm{cm}^{2} \mathrm{~V}^{-1} \mathrm{~s}^{-1}$ and $10^{2}-10^{3}$, respectively, but after oxidation, 90 and $95 \%$ reductions have been reported for hole mobility and $I_{\mathrm{ON}} / I_{\mathrm{OFF}}$ ratios, respectively. ${ }^{30,52}$ In more detail, it has been identified that oxygen exposure may affect FET devices in a two-step process: first, during oxygen physisorption (i.e., before chemical oxidation), only electron mobility is suppressed, but next, after chemical oxidation (light assisted), significant hole and electron mobility reductions were also reported. $^{53}$

In summary, the complete picture of few-layer black phosphorus oxidation can be presented as follows. First, surface-oxidized few-layer black phosphorus with low oxygen concentration per unit cell is formed during sonication and sample manipulation. It is present at temperatures below 373 $K$. During the endothermic and mass transition in the temperature range 373 to $448 \mathrm{~K}$, the material most likely contains a mixed phase of planar and surface oxides and higher oxygen concentration, when available. Finally, at temperatures above $448 \mathrm{~K}$, the interstitial oxides are the most stable forms with measurable consequences in lattice deformation ${ }^{23}$ and the electronic structure (Figure 6). This presents unexpected opportunities for electric device engineering using interstitial phosphorene oxide, as interstitial oxides exhibit unique trends responsible for doubling the band gap while decreasing the work function of exfoliated black phosphorus. At the end of the endothermic process, the ohmic contact is due to a better band alignment where charge carriers can flow without potential barrier height (or minimal) between the gold electrode and the oxidized few-layer BP.

\section{CONCLUSIONS}

Bandgap engineering by native oxides has been proposed recently in theoretical works. ${ }^{21}$ Here, we present the first experimental evidence that interstitial oxides may indeed improve the band alignment between $\mathrm{Au}$ electrodes and exfoliated black phosphorus for transducer applications. Our computational calculations evidence a monotonic increase of the work function with the oxygen concentration at the dangling position explaining the increased electrical resistance in electric devices used recently as the indicator of degradation. Moreover, a dangling-to-interstitial oxide transition abruptly modifies the lattice and electronic structure of the material between 373 and $448 \mathrm{~K}$.

The formation of metastable black phosphorus oxides has been reported for liquid-phase exfoliated materials. ${ }^{43,44}$ Here, new evidence on the relative stability of the dangling and interstitial oxide at room temperature has been presented. The surface oxides are the primary outcome of the few-layer black phosphorus oxidation, and the transition to the planar configuration requires additional energy. The dangling-tointerstitial transition starts at $373 \mathrm{~K}$ in agreement with the thermally activated electric conduction measurements and previous results. ${ }^{23}$ This temperature for the phosphorene oxides transition is an operational limit to be considered in future applications of exfoliated black phosphorus, as the electronic structure is modified and can alter the response of devices, for example, in transducer applications.

A secondary outcome of the present work is that the activation energy for thermally activated electric conduction is a good quantitative descriptor of oxidation processes. Since measuring electrical resistance as a function of temperature is much more feasible than some other methods previously utilized for monitoring oxidation processes (e.g., Raman spectroscopy and thermogravimetry), we propose that this methodology could be useful for the evaluation of passivated/ functionalized few-layer black phosphorus. The dangling-tointerstitial transition would be possible only in the presence of small dangling substituents less affected by steric effects (i.e., dangling oxygen in this case). The absence of such substituents (i.e., pristine few-layer black phosphorus) would result in a lattice thermal expansion without the deformations measured for the out-of-plane vibrations detected with in situ Raman spectroscopy. ${ }^{23}$ The activation energy for thermally activated electric conduction for semiconducting surface oxides (79 $\mathrm{meV})$ was $\sim 40$ times lower than that for semiconducting planar oxides $(3295 \mathrm{meV})$.

\section{ASSOCIATED CONTENT}

\section{Supporting Information}

The Supporting Information is available free of charge at https://pubs.acs.org/doi/10.1021/acs.jpcc.0c06542. 
Basic characterization and activation energy data for thermally activated electric conduction for comparison and verification purposes; current-voltage characteristics in an oxygen-rich atmosphere and multilayer samples for comparison and verification; and DOS variations with the oxygen concentration at dangling and interstitial positions (PDF)

Atomic coordinates for all structures in format XSF (ZIP)

\section{AUTHOR INFORMATION}

\section{Corresponding Authors}

Juan F. Gómez-Pérez - Interdisciplinary Excellence Centre, Department of Applied and Environmental Chemistry, University of Szeged, H-6720 Szeged, Hungary; 이이. ord/ 0000-0002-2736-2015; Email: juan.gomez@chem.uszeged.hu

Ákos Kukovecz - Interdisciplinary Excellence Centre, Department of Applied and Environmental Chemistry, University of Szeged, H-6720 Szeged, Hungary; 이이.org/ 0000-0003-0716-9557; Email: kakos@chem.u-szeged.hu

\section{Authors}

Julián D. Correa - Universidad de Medellin, Facultad de Ciencias Básicas, 050026, Colombia

Cora Bartus Pravda - Interdisciplinary Excellence Centre, Department of Applied and Environmental Chemistry, University of Szeged, H-6720 Szeged, Hungary

Zoltán Kónya - Interdisciplinary Excellence Centre, Department of Applied and Environmental Chemistry and MTA-SZTE Reaction Kinetics and Surface Chemistry Research Group, University of Szeged, H-6720 Szeged, Hungary; 이이.org/ 0000-0002-9406-8596

Complete contact information is available at:

https://pubs.acs.org/10.1021/acs.jpcc.0c06542

\section{Notes}

The authors declare no competing financial interest.

\section{ACKNOWLEDGMENTS}

Financial support from the Hungarian National Research, Development, and Innovation Office through projects GINOP-2.3.2-15-2016-00013 'Intelligent materials based on functional surfaces - from syntheses to applications', K120115 (ZK), and K126065 (ÁK) is acknowledged. J.G.P. acknowledges the financial support from the Administrative Department of Science, Technology, and Innovation of Colombia (Colciencias). J.D.C. thanks MINCIENCIAS for the financial support of this research by contract 120680864729 .

\section{REFERENCES}

(1) Buscema, M.; Groenendijk, D. J.; Blanter, S. I.; Steele, G. A.; Van Der Zant, H. S. J.; Castellanos-Gomez, A. Fast and broadband photoresponse of few-layer black phosphorus field-effect transistors. Nano Lett. 2014, 14, 3347-3352.

(2) Abbas, A. N.; Liu, B.; Chen, L.; Ma, Y.; Cong, S.; Aroonyadet, N.; Köpf, M.; Nilges, T.; Zhou, C. Black phosphorus gas sensors. ACS Nano 2015, 9, 5618-5624.

(3) Cui, S.; Pu, H.; Wells, S. A.; Wen, Z.; Mao, S.; Chang, J.; Hersam, M. C.; Chen, J. Ultrahigh sensitivity and layer-dependent sensing performance of phosphorene-based gas sensors. Nat. Commun. 2015, 6, 8632.
(4) Hanlon, D.; et al. Liquid exfoliation of solvent-stabilized fewlayer black phosphorus for applications beyond electronics. Nat. Commun. 2015, 6, 8563.

(5) Yasaei, P.; Kumar, B.; Foroozan, T.; Wang, C.; Asadi, M.; Tuschel, D.; Indacochea, J. E.; Klie, R. F.; Salehi-Khojin, A. HighQuality Black Phosphorus Atomic Layers by Liquid-Phase Exfoliation. Adv. Mater. 2015, 27, 1887-1892.

(6) Chen, Y.; Ren, R.; Pu, H.; Chang, J.; Mao, S.; Chen, J. Fieldeffect transistor biosensors with two-dimensional black phosphorus nanosheets. Biosens. Bioelectron. 2017, 89, 505-510.

(7) Lv, Y.; Qin, W.; Wang, C.; Liao, L.; Liu, X. Recent Advances in Low-Dimensional Heterojunction-Based Tunnel Field Effect Transistors. Adv. Electron. Mater. 2018, 5, 1800569.

(8) Bai, L.; et al. Black Phosphorus/Platinum Heterostructure: A Highly Efficient Photocatalyst for Solar-Driven Chemical Reactions. Adv. Mater. 2018, 30, 1803641.

(9) Zhang, G.; Huang, S.; Chaves, A.; Song, C.; Özçelik, V. O.; Low, T.; Yan, H. Infrared fingerprints of few-layer black phosphorus. Nat. Commun. 2017, 8, 14071.

(10) Li, L.; Yu, Y.; Ye, G. J.; Ge, Q.; Ou, X.; Wu, H.; Feng, D.; Chen, X. H.; Zhang, Y. Black phosphorus field-effect transistors. Nat. Nanotechnol. 2014, 9, 372-377.

(11) Novoselov, K. S.; Geim, A. K.; Morozov, S. V.; Jiang, D.; Katsnelson, M. I.; Grigorieva, I. V.; Dubonos, S. V.; Firsov, A. A. Twodimensional gas of massless Dirac fermions in graphene. Nature 2005, 438, 197-200.

(12) Bolotin, K. I.; Sikes, K. J.; Jiang, Z.; Klima, M.; Fudenberg, G.; Hone, J.; Kim, P.; Stormer, H. L. Ultrahigh electron mobility in suspended graphene. Solid State Commun. 2008, 146, 351-355.

(13) Yu, Z.; Ong, Z. Y.; Li, S.; Xu, J.; Bin, Z. G.; Zhang, Y. W.; Shi, Y.; Wang, X. Analyzing the Carrier Mobility in Transition-Metal Dichalcogenide MoS2 Field-Effect Transistors. Adv. Funct. Mater. 2017, 27, 1604093.

(14) Ziletti, A.; Carvalho, A.; Campbell, D. K.; Coker, D. F.; Castro Neto, A. H. Oxygen defects in phosphorene. Phys. Rev. Lett. 2015, $114,26-29$.

(15) Wang, G.; Pandey, R.; Karna, S. P. Phosphorene Oxide: Stability and Electronic Properties of a Novel Two-Dimensional Material. Nanoscale 2015, 7, 524-531.

(16) Wang, J.; Wei, L.; Zhang, L.; Jiang, C.; Siu-Wai Kong, E.; Zhang, Y. Preparation of high aspect ratio nickel oxide nanowires and their gas sensing devices with fast response and high sensitivity. J. Mater. Chem. 2012, 22, 8327.

(17) Kc, S.; Longo, R. C.; Wallace, R. M.; Cho, K. Surface oxidation energetics and kinetics on MoS2 monolayer. J. Appl. Phys. 2015, 117, 135301.

(18) Zhao, Y.; Wu, X.; Yang, J.; Zeng, X. C. Oxidation of a twodimensional hexagonal boron nitride monolayer: A first-principles study. Phys. Chem. Chem. Phys. 2012, 14, 5545-5550.

(19) Van Druenen, M.; Davitt, F.; Collins, T.; Glynn, C.; O'Dwyer, C.; Holmes, J. D.; Collins, G. Covalent Functionalization of FewLayer Black Phosphorus Using Iodonium Salts and Comparison to Diazonium Modified Black Phosphorus. Chem. Mater. 2018, 30, 4667-4674.

(20) Ryder, C. R.; Wood, J. D.; Wells, S. A.; Yang, Y.; Jariwala, D.; Marks, T. J.; Schatz, G. C.; Hersam, M. C. Covalent functionalization and passivation of exfoliated black phosphorus via aryl diazonium chemistry. Nat. Chem. 2016, 8, 597-602.

(21) Ziletti, A.; Carvalho, A.; Trevisanutto, P. E.; Campbell, D. K.; Coker, D. F.; Castro, N. A. H. Phosphorene oxides: Bandgap engineering of phosphorene by oxidation. Phys. Rev. B 2015, 91, No. 085407.

(22) Wild, S.; et al. Monolayer black phosphorus by sequential wetchemical surface oxidation. RSC Adv. 2019, 9, 3570-3576.

(23) Gómez-Pérez, J.; Barna, B.; Tóth, I. Y.; Kónya, Z.; Kukovecz, Á. Quantitative tracking of the oxidation of black phosphorus in the fewlayers regime. ACS Omega 2018, 3, 12482-12488. 
(24) Wild, S.; et al. Lattice Opening Upon Bulk Reductive Covalent Functionalization of Black Phosphorus. Angew. Chem., Int. Ed. 2019, 58, 5763-5768.

(25) Marcia, M.; Hirsch, A.; Hauke, F. Perylene-based non-covalent functionalization of 2D materials. FlatChem 2017, 1, 89-103.

(26) Edmonds, M. T.; et al. Creating a stable oxide at the surface of black phosphorus. ACS Appl. Mater. Interfaces 2015, 7, 14557-14562.

(27) Wang, C.-X.; Zhang, C.; Jiang, J.-W.; Rabczuk, T. The Effects of Vacancy and Oxidation on Black Phosphorus Nanoresonators. Nanotechnology 2017, 28, 135202.

(28) Wang, G.; Slough, W. J.; Pandey, R.; Karna, S. P. Degradation of phosphorene in air: understanding at atomic level. 2D Mater. 2016, 3, No. 025011.

(29) Kuntz, K. L.; et al. Control of Surface and Edge Oxidation on Phosphorene. ACS Appl. Mater. Interfaces 2017, 9, 9126-9135.

(30) Lv, W.; et al. Sulfur-Doped Black Phosphorus Field-Effect Transistors with Enhanced Stability. ACS Appl. Mater. Interfaces 2018, 10, 9663-9668.

(31) Hsieh, Y. L.; Su, W. H.; Huang, C. C.; Su, C. Y. In Situ Cleaning and Fluorination of Black Phosphorus for Enhanced Performance of Transistors with High Stability. ACS Appl. Mater. Interfaces 2020, 12, 37375-37383.

(32) Su, C.; et al. Waterproof molecular monolayers stabilize 2D materials. Proc. Natl. Acad. Sci. U. S. A. 2019, 116, 20844-20849.

(33) Cai, Y.; Zhang, G.; Zhang, Y.-W. Layer-dependent band alignment and work function of few-layer phosphorene. Sci. Rep. 2014, 4, 6677.

(34) Castellanos-Gomez, A.; et al. Isolation and characterization of few-layer black phosphorus. 2D Mater. 2014, 1, No. 025001.

(35) Island, J. O.; Steele, G. A.; Van Der Zant, H. S. J.; CastellanosGomez, A. Environmental instability of few-layer black phosphorus. 2D Mater. 2015, 2, 011002.

(36) Wood, J. D.; et al. Effective Passivation of Exfoliated Black Phosphorus Transistors against Ambient Degradation. Nano Lett. 2014, 14, 6964-6970.

(37) Gómez-Pérez, J.; Kónya, Z.; Kukovecz, Á. Acetone improves the topographical homogeneity of liquid phase exfoliated few-layer black phosphorus flakes. Nanotechnology 2018, 29, 365303.

(38) Soler, J. M.; Artacho, E.; Gale, J. D.; García, A.; Junquera, J.; Ordejón, P.; Sánchez-Portal, D. The SIESTA method for ab initio order-N materials simulation. J. Phys. Condens. Matter 2002, 14, 2745-2779.

(39) Perdew, J. P.; Burke, K.; Ernzerhof, M. Generalized gradient approximation made simple. Phys. Rev. Lett. 1996, 77, 3865-3868.

(40) Woomer, A. H.; Farnsworth, T. W.; Hu, J.; Wells, R. A.; Donley, C. L.; Warren, S. C. Phosphorene: Synthesis, Scale-Up, and Quantitative Optical Spectroscopy. ACS Nano 2015, 9, 8869-8884.

(41) Favron, A.; et al. Photooxidation and quantum confinement effects in exfoliated black phosphorus. Nat. Mater. 2015, 14, 826-832.

(42) Abellán, G.; et al. Fundamental Insights into the Degradation and Stabilization of Thin Layer Black Phosphorus. J. Am. Chem. Soc. 2017, 139, 10432-10440.

(43) Brent, J. R.; Ganguli, A. K.; Kumar, V.; Lewis, D. J.; McNaughter, P. D.; O’Brien, P.; Sabherwal, P.; Tedstone, A. A. On the stability of surfactant-stabilised few-layer black phosphorus in aqueous media. RSC Adv. 2016, 6, 86955-86958.

(44) Gamage, S.; Li, Z.; Yakovlev, V. S.; Lewis, C.; Wang, H.; Cronin, S. B.; Abate, Y. Nanoscopy of Black Phosphorus Degradation. Adv. Mater. Interfaces 2016, 3, 1600121.

(45) Quintanilla, J.; Hooley, C. The strong-correlations puzzle. Phys. World 2009, 22, 32-37.

(46) Sibari, A.; et al. Phosphorene as a promising anode material for (Li/ $\mathrm{Na} / \mathrm{Mg}$ )-ion batteries: A first-principle study. Sol. Energy Mater. Sol. Cells 2018, 180, 253-257.

(47) Mortazavi, B.; Dianat, A.; Cuniberti, G.; Rabczuk, T. Application of silicene, germanene and stanene for $\mathrm{Na}$ or $\mathrm{Li}$ ion storage: A theoretical investigation. Electrochim. Acta 2016, 213, 865870 .
(48) Wang, X.; Liu, G.; Liu, R. F.; Luo, W. W.; Sun, B. Z.; Lei, X. L.; Ouyang, C. Y.; Xu, B. Molecular adsorption and strain-induced ferromagnetic semiconductor-metal transition in half-hydrogenated germanene. J. Appl. Phys. 2019, 125, 082504.

(49) Kim, M.; Kim, H.; Park, S.; Kim, J. S.; Choi, H. J.; Im, S.; Lee, H.; Kim, T.; Yi, Y. Intrinsic correlation between electronic structure and degradation: from few layers to bulk black phosphorus. Angew. Chem., Int. Ed. 2019, 58, 3754-3758.

(50) Greiner, M. T.; Chai, L.; Helander, M. G.; Tang, W. M.; Lu, Z. $\mathrm{H}$. Transition metal oxide work functions: The influence of cation oxidation state and oxygen vacancies. Adv. Funct. Mater. 2012, 22, 4557-4568.

(51) Fijol, J. F.; Holloway, P. H. Ohmic contacts to ZnSe-based materials. Crit. Rev. Solid State Mater. Sci. 1996, 21, 77-128.

(52) Yang, B.; et al. Te-Doped Black Phosphorus Field-Effect Transistors. Adv. Mater. 2016, 28, 9408-9415.

(53) Han, C.; et al. Oxygen induced strong mobility modulation in few-layer black phosphorus. 2D Mater. 2017, 4, 021007. 